\title{
Clinical Study \\ Preoperative Evaluation with fMRI of Patients with Intracranial Gliomas
}

\author{
Ioannis Z. Kapsalakis, ${ }^{1}$ Eftychia Z. Kapsalaki, ${ }^{2}$ Efstathios D. Gotsis, ${ }^{3}$ \\ Dimitrios Verganelakis, ${ }^{3}$ Panagiotis Toulas, ${ }^{3}$ Georgios Hadjigeorgiou, ${ }^{1}$ Indug Chung, ${ }^{4}$ \\ Ioannis Fezoulidis, ${ }^{2}$ Alexandros Papadimitriou, ${ }^{1}$ Joe Sam Robinson, ${ }^{4}$ \\ Gregory P. Lee, ${ }^{5}$ and Kostas N. Fountas ${ }^{6,7}$
}

${ }^{1}$ Department of Neurology, School of Medicine, University Hospital of Larisa, University of Thessaly, 41110 Larisa, Greece

${ }^{2}$ Department of Diagnostic Radiology, School of Medicine, University Hospital of Larisa, University of Thessaly, 41110 Larisa, Greece

${ }^{3}$ Department of MR Imaging, Advanced Diagnostic and Research Institute "Euromedica-Encephalos", 15233 Athens, Greece

${ }^{4}$ Departments of Neurosurgery and Intraoperative Electrophysiology, Medical Center of Central Georgia, School of Medicine, Mercer University, Macon, GA 31201, USA

${ }^{5}$ Department of Neurology, Medical College of Georgia, Augusta, GA 30912, USA

${ }^{6}$ Department of Neurosurgery, School of Medicine, University Hospital of Larisa, University of Thessaly, 41110 Larisa, Greece

${ }^{7}$ Institute of Biomedical Research and Technology (BIOMED), Center for Research and Technology-Thessaly (CERETETH), 38500 Larissa, Greece

Correspondence should be addressed to Kostas N. Fountas, knfountasmd@excite.com

Received 20 March 2012; Revised 1 May 2012; Accepted 7 May 2012

Academic Editor: Kyousuke Kamada

Copyright (C) 2012 Ioannis Z. Kapsalakis et al. This is an open access article distributed under the Creative Commons Attribution License, which permits unrestricted use, distribution, and reproduction in any medium, provided the original work is properly cited.

Introduction. Aggressive surgical resection constitutes the optimal treatment for intracranial gliomas. However, the proximity of a tumor to eloquent areas requires exact knowledge of its anatomic relationships to functional cortex. The purpose of our study was to evaluate fMRI's accuracy by comparing it to intraoperative cortical stimulation (DCS) mapping. Material and Methods. Eightyseven patients, with presumed glioma diagnosis, underwent preoperative fMRI and intraoperative DCS for cortical mapping during tumor resection. Findings of fMRI and DCS were considered concordant if the identified cortical centers were less than $5 \mathrm{~mm}$ apart. Pre and postoperative Karnofsky Performance Scale and Spitzer scores were recorded. A postoperative MRI was obtained for assessing the extent of resection. Results. The areas of interest were identified by fMRI and DCS in all participants. The concordance between fMRI and DCS was $91.9 \%$ regarding sensory-motor cortex, $100 \%$ for visual cortex, and $85.4 \%$ for language. Data analysis showed that patients with better functional condition demonstrated higher concordance rates, while there also was a weak association between tumor grade and concordance rate. The mean extent of tumor resection was $96.7 \%$. Conclusions. Functional MRI is a highly accurate preoperative methodology for sensory-motor mapping. However, in language mapping, DCS remains necessary for accurate localization.

\section{Introduction}

Resection of brain tumors involving eloquent cortical areas has remained a challenging task [1-6]. Preservation of neuronal functions after surgery remains the goal for patients with primary and/or metastatic tumors involving the central, visual, Broca's, and/or Wernicke's areas. Intraoperative electric direct cortical stimulation (DCS) and mapping can accurately identify and define eloquent cortical areas, can examine their spatial relationships with the tumor, and can facilitate aggressive tumor resection $[3,4,7-11]$. However, DCS mapping requires either an awake craniotomy and a cooperative patient, at least for language area mapping, or a second operative procedure for extraoperative cortical 
stimulation and mapping via previously implanted subdural electrodes [4, 12-15]. In addition, DCS can identify cortical language-associated areas but cannot easily outline subcortical or intrasulcal speech areas $[16,17]$.

Functional MRI (fMRI) is a noninvasive, imaging modality that has been used for mapping regions of the brain associated with motor, sensory, language, vision, and other cognitive tasks [18-25]. The change in signal detected by fMRI as neuronal activation is presumed to result from changes in regional, temporary concentrations of oxyhemoglobin caused by increased regional blood flow [26]. It has been extensively described that fMRI is based on a complex physiological phenomenon called Blood-OxygenationLevel-Dependent (BOLD) effect [26]. The employment of fMRI in the presurgical planning of patients with brain tumors adjacent or in eloquent cortical areas has been increasing, in order to minimize the possibility of postoperative neurological deficit while may maximize the extent of tumor resection [6, 27-59]. An exponentially increasing number of clinical investigators have been applying various fMRI paradigms and protocols in patients with primary or metastatic tumors for identifying preoperatively their relationship with eloquent cortical areas [6, 27-59]. However, there is a significant variation in the reported fMRI accuracy rates, and frequently conflicting conclusions regarding the value of $\mathrm{fMRI}$ in the preoperative evaluation of patients with intracranial tumors [6, 27-59].

In our current study, we present our results from employing both preoperative fMRI and intraoperative electric DCS on patients undergoing craniotomy for supratentorial glioma resection. The accuracy of the preoperative fMRI was compared to the intraoperative electrophysiologic findings, and the overall role of fMRI in intracranial glioma surgery was evaluated.

\section{Material and Methods}

Our prospective clinical study covered a 10-year period (2002-2011). The study was approved by the Institutional Review Board of all the participating institutions. A signed written consent form was obtained from the participants or their legal representatives. The analysis of our data was performed according to the regulations of the current Health Insurance Portability and Accountability Act.

The inclusion criteria in our study were (i) patient's age $>18$ years, (ii) presence of novel, supratentorial, presumably glial tumor (based on conventional MRI and proton MR spectroscopy whenever available), (iii) tumor location within or in the close proximity of eloquent cortical area, and (iv) patient's consent to participate in the study protocol. Patients with recurrent tumors, or previously irradiated tumors, were excluded from our study.

A total of 892 patients with supratentorial tumors were evaluated during the study period in the participating institutions. However, only 87 patients (53 males and 34 females) met our inclusion criteria and participated in our study (Table 1). Their ages ranged between 33 and 76 years

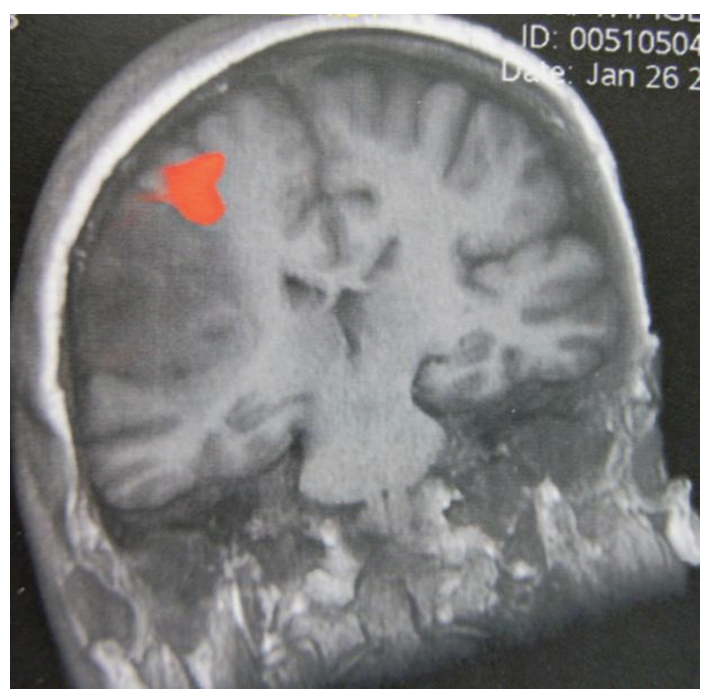

FIGURE 1: fMRI showing right hand activation antero-superiorly to the lesion.

(mean age: 62.8). Detailed pre and postoperative neurological examinations, as well as neuropsychological (Mini Mental Status Examination), performance scores (Karnofsky Performance Scale, KPS, scores), and quality of daily life (Spitzer Quality of Life Index) evaluations were obtained in all cases. The postoperative KPS, and Spitzer indices were obtained one month after the patients' discharge.

A conventional brain MRI study in a $1.5 \mathrm{~T}$ MRI scanner was obtained in all patients. An fMRI study was also obtained in all participants. The study was obtained within a month prior to their scheduled surgery. Foam cushions and straps were used for comfortably immobilizing the patient's head. BRAVO pulse sequence was utilized for obtaining the 3dimensional anatomical images (248 images, flip angle $=15^{\circ}$, $\mathrm{TE}=3.7 \mathrm{~ms}, \mathrm{TR}=9 \mathrm{~ms}$, Th0/Sp $=1.4 /-0.7 \mathrm{~mm}$, FOV: $26 \times 26)$. The following motor tasks were used: (i) a fingerthumb tapping test, (ii) periodic fist clenching/spreading test, (iii) periodic active movement of the foot, and (iv) periodic circular movements of the tongue (Figure 1). Tactile stimulation of the face, the hand, and/or the foot with a toothbrush was used for identifying the postcentral gyrus. The following verbal tasks were used for identifying the language associated cortical areas: (i) picture naming, (ii) word listening and parroting also, (iii) production of a noun from a verb or vice versa, (iv) finding a word of the opposite meaning in a given word, (v) reciting a well-known poem or a song, (iv) performance of simple mathematical calculations, (vii) countdown from 100 by subtracting 7 . Each task performance test lasted 2.5 min including two periods of activation interspersed with three periods of rest of $30 \mathrm{sec}$ duration each. In addition, a head CT scan was also obtained the day before the procedure for image fusion purposes and for ruling out the possibility of magnetic image distortion.

A StealthStation S7 neuro-navigational system (Medtronic, Minneapolis, MN, USA) was used in all our cases. The MRI, fMRI, and CT scans of each patient were uploaded, 


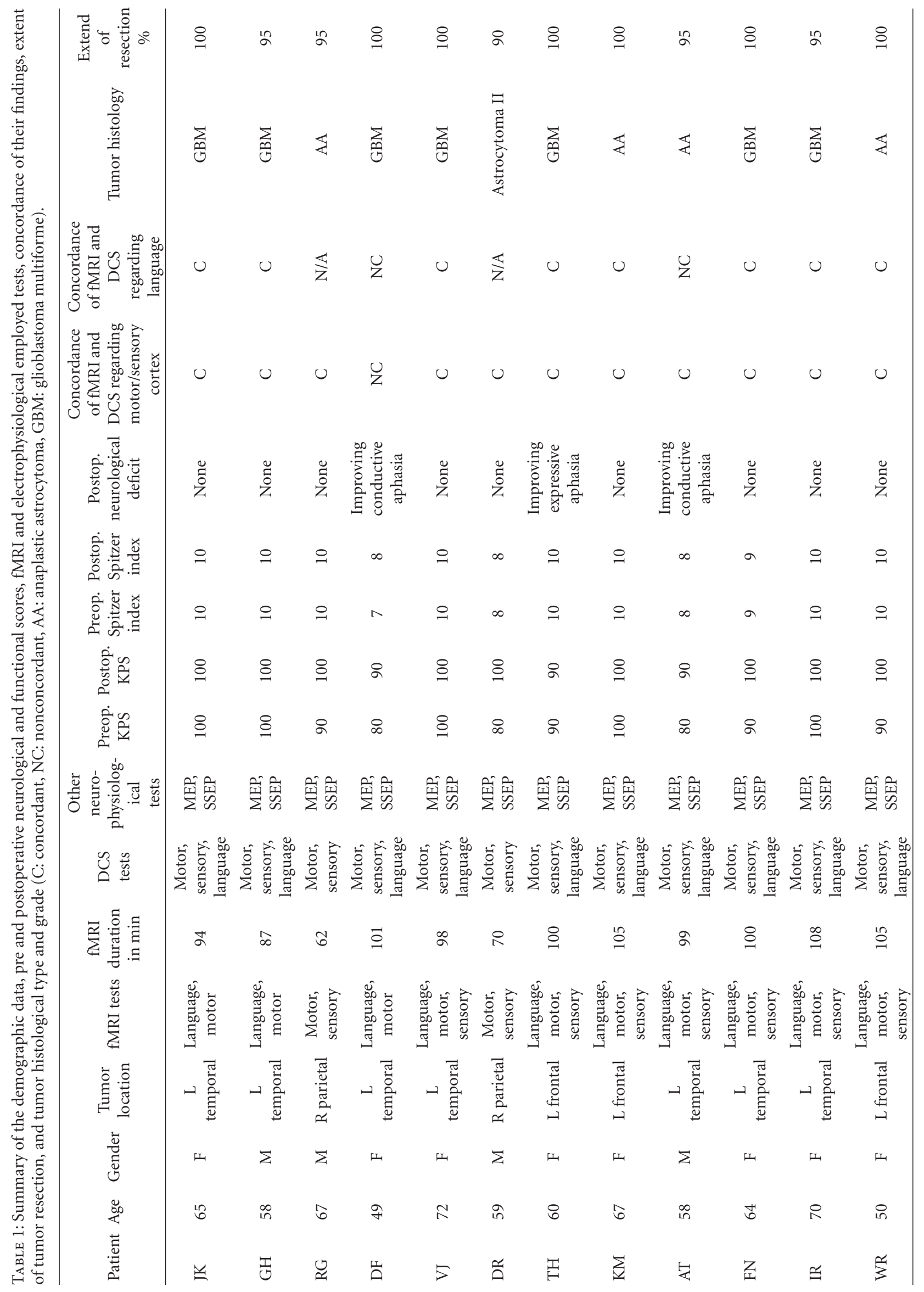




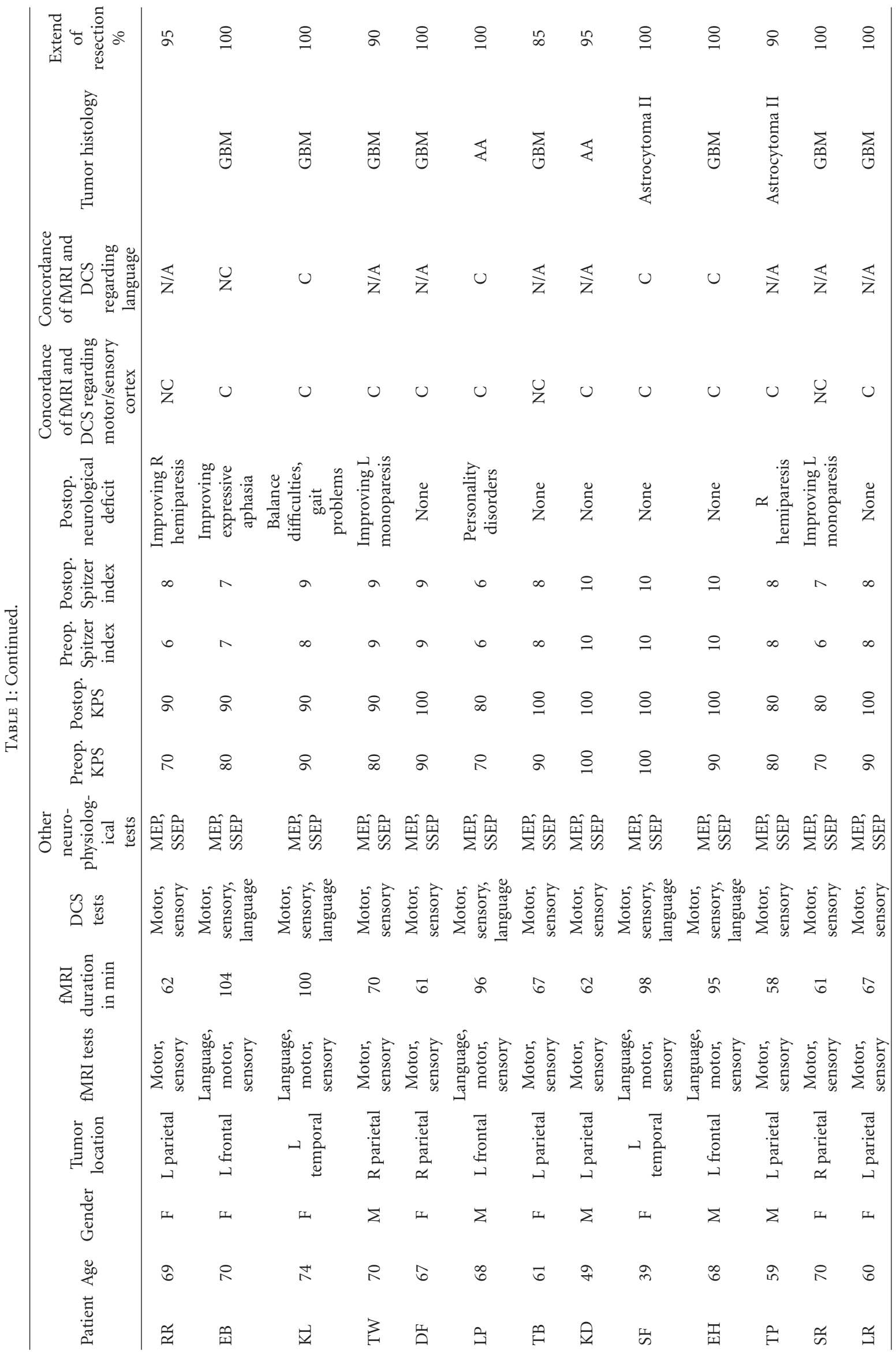




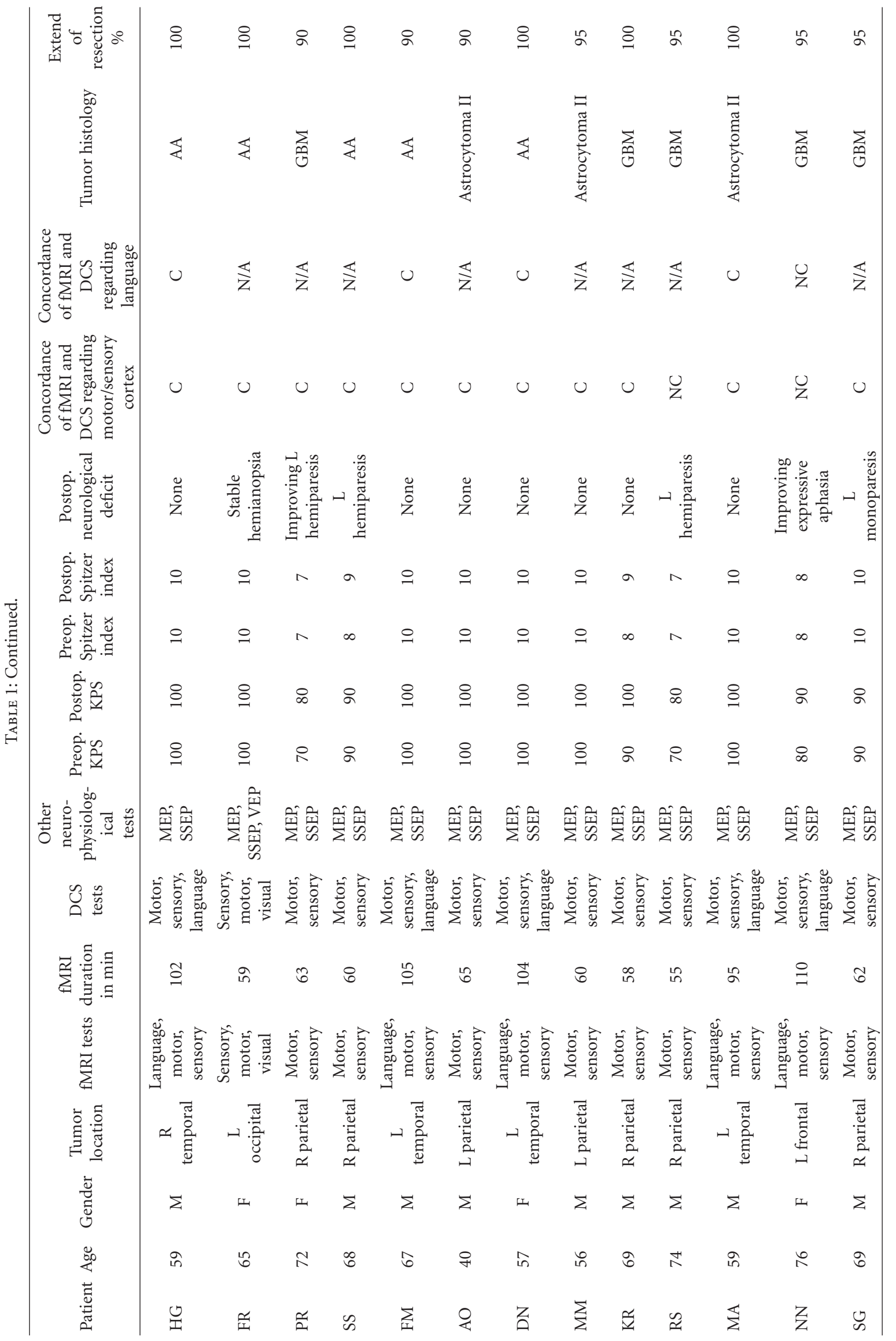




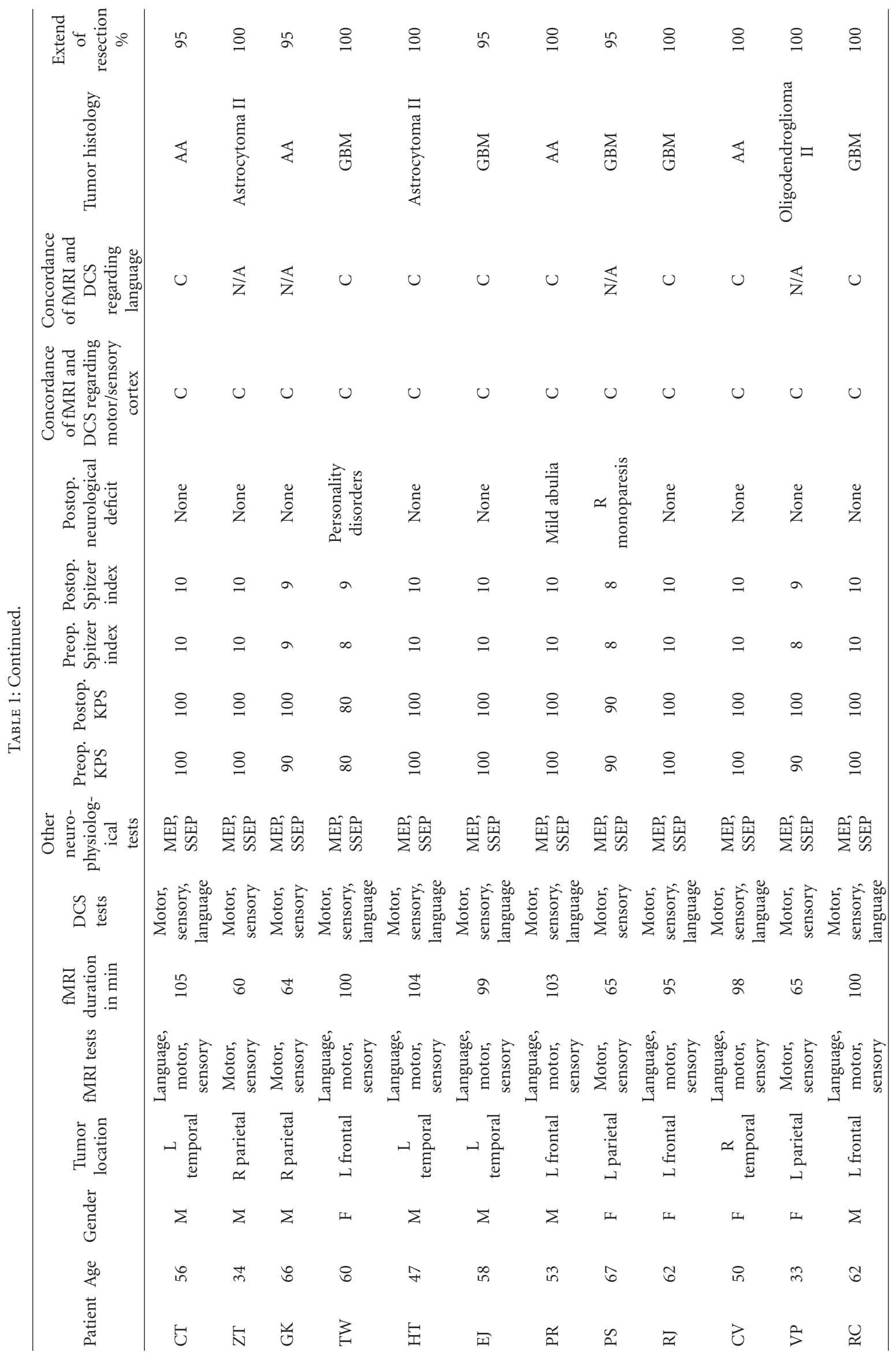




\begin{tabular}{|c|c|c|c|c|c|c|c|c|c|c|c|c|}
\hline 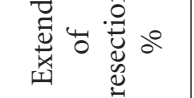 & ฉ & 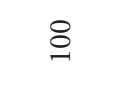 & 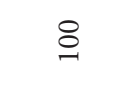 & 으 & 느 & นn & $\stackrel{-}{\circ}$ & 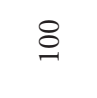 & 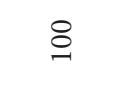 & $\stackrel{2}{\alpha}$ & $\stackrel{8}{ }$ & 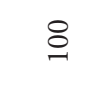 \\
\hline 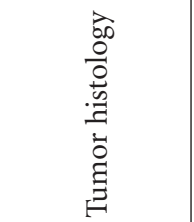 & $\sum_{00}^{0}$ & 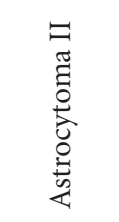 & 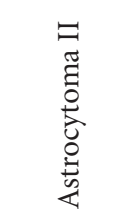 & 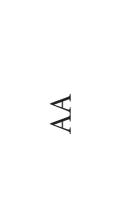 & $\mathbb{4}$ & $\mathbb{4}$ & $\sum_{\text {焉 }}$ & $\sum_{\substack{0 \\
0}}$ & $\mathbb{4}$ & \& & $\sum_{\substack{0 \\
0}}^{\sum}$ & $\sum_{\substack{\infty \\
心}}$ \\
\hline 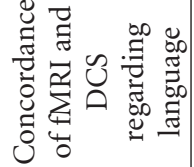 & $\overleftrightarrow{\mathrm{z}}$ & u & $u$ & $u$ & $\underset{\mathrm{z}}{\overleftrightarrow{\mathrm{z}}}$ & $u$ & $u$ & $\underset{\mathrm{Z}}{\overleftrightarrow{\mathrm{z}}}$ & $u$ & $\underset{\mathrm{Z}}{\overleftrightarrow{\mathrm{z}}}$ & U & $u$ \\
\hline 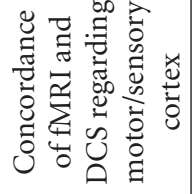 & z & $\cup$ & $\cup$ & $u$ & $u$ & $u$ & $u$ & $u$ & $u$ & $u$ & u & u \\
\hline 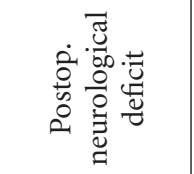 & 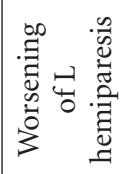 & Z̃ & 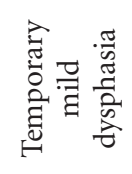 & ஜ̆ & 气̆ & 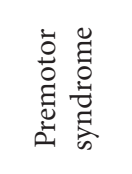 & 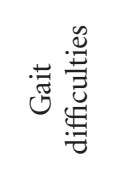 & 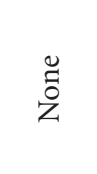 & ஜ̈ & 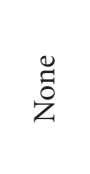 & 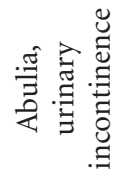 & 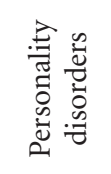 \\
\hline 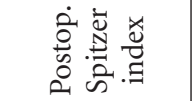 & $\infty$ & 으 & $\stackrel{\circ}{\circ}$ & 으 & $ㅇ$ & $\stackrel{\circ}{\circ}$ & $a$ & $\stackrel{\circ}{ }$ & $\circ$ & $ㅇ$ & $\infty$ & $ㅇ$ \\
\hline 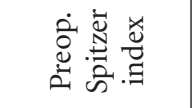 & $\wedge$ & 으 & $\stackrel{0}{ }$ & 으 & 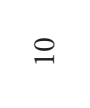 & 으 & $\infty$ & $\circ$ & 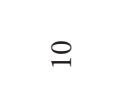 & 으 & $\infty$ & $\stackrel{ }{2}$ \\
\hline 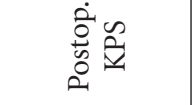 & $\stackrel{R}{R}$ & $\nsubseteq$ & 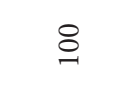 & 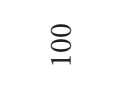 & 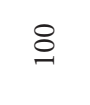 & $\stackrel{-}{\circ}$ & \& & 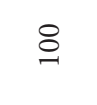 & \& & \& & \& & $\stackrel{0}{ }$ \\
\hline 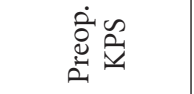 & $\infty$ & $\stackrel{8}{\circ}$ & 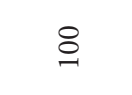 & 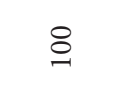 & $\stackrel{8}{\circ}$ & $\stackrel{8}{\circ}$ & \& & $\stackrel{8}{0}$ & 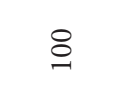 & $\stackrel{8}{\circ}$ & \& & $\stackrel{8}{ }$ \\
\hline 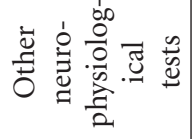 & $\begin{array}{l}\hat{y} \\
\dot{\Sigma}\end{array}$ & 氙悹 & 義语 & 密岭 & 窐昰 & 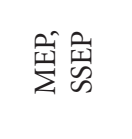 & 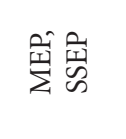 & 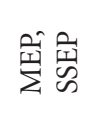 & 氙跮 & 窐嘧 & 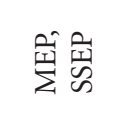 & 畕窇 \\
\hline U⿺辶巛 & 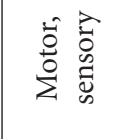 & 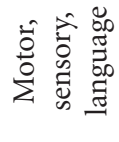 & 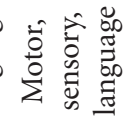 & 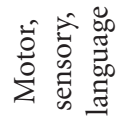 & 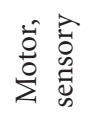 & 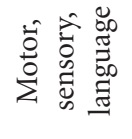 & 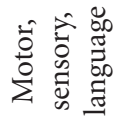 & 竞总 & 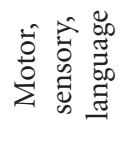 & 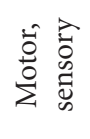 & 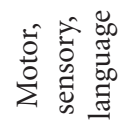 & 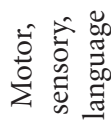 \\
\hline 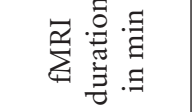 & $\stackrel{\infty}{i n}$ & తి & 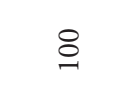 & $\stackrel{\infty}{\circ}$ & in & తి & 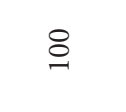 & $\stackrel{\infty}{i n}$ & นn & 8 & $\underset{-}{+}$ & $\stackrel{2}{\alpha}$ \\
\hline 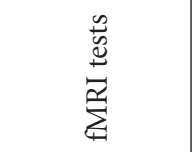 & 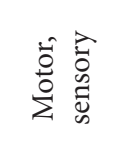 & 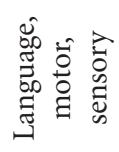 & 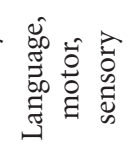 & 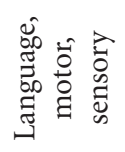 & 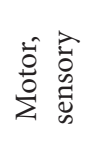 & 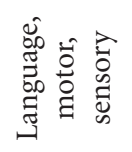 & 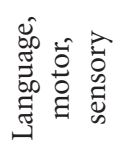 & 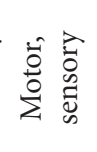 & 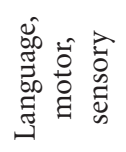 & 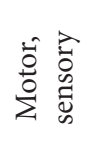 & 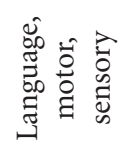 & 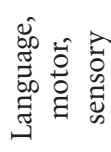 \\
\hline 藏 & 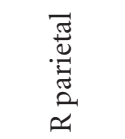 & 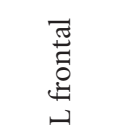 & • & 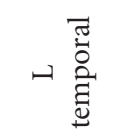 & 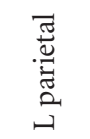 & 苞 & ↔ & 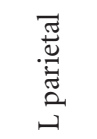 & 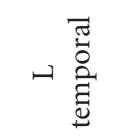 & 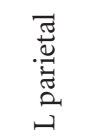 & 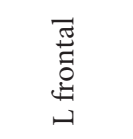 & 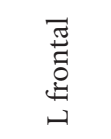 \\
\hline 节 & 山 & $\Sigma$ & $\Sigma$ & $\Sigma$ & $\Sigma$ & $\Sigma$ & $\Sigma$ & $\Sigma$ & 山 & $\Sigma$ & $\Sigma$ & $\Sigma$ \\
\hline$\underset{8}{80}$ & \$ & i & 8 & in & $\hat{\sigma}$ & i & 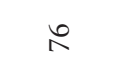 & $R$ & in & in & $\therefore$ & oి \\
\hline 䔍 & $\ddot{b}$ & 安 & $\frac{x}{3}$ & $\stackrel{\mathbb{I}}{\Omega}$ & 光 & 䍐 & Z & \& & $\infty$ & 岂 & 光 & 4 \\
\hline
\end{tabular}




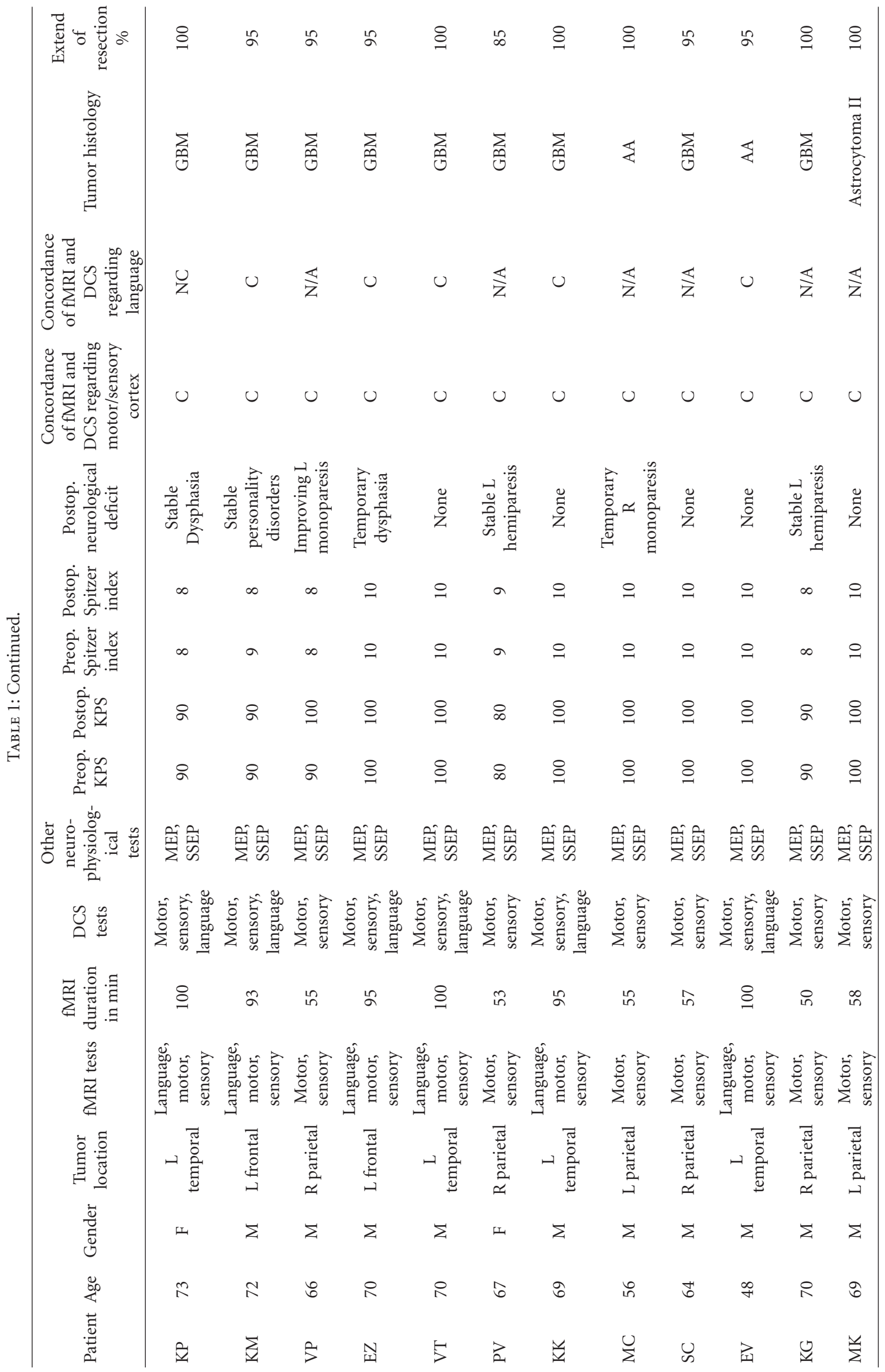




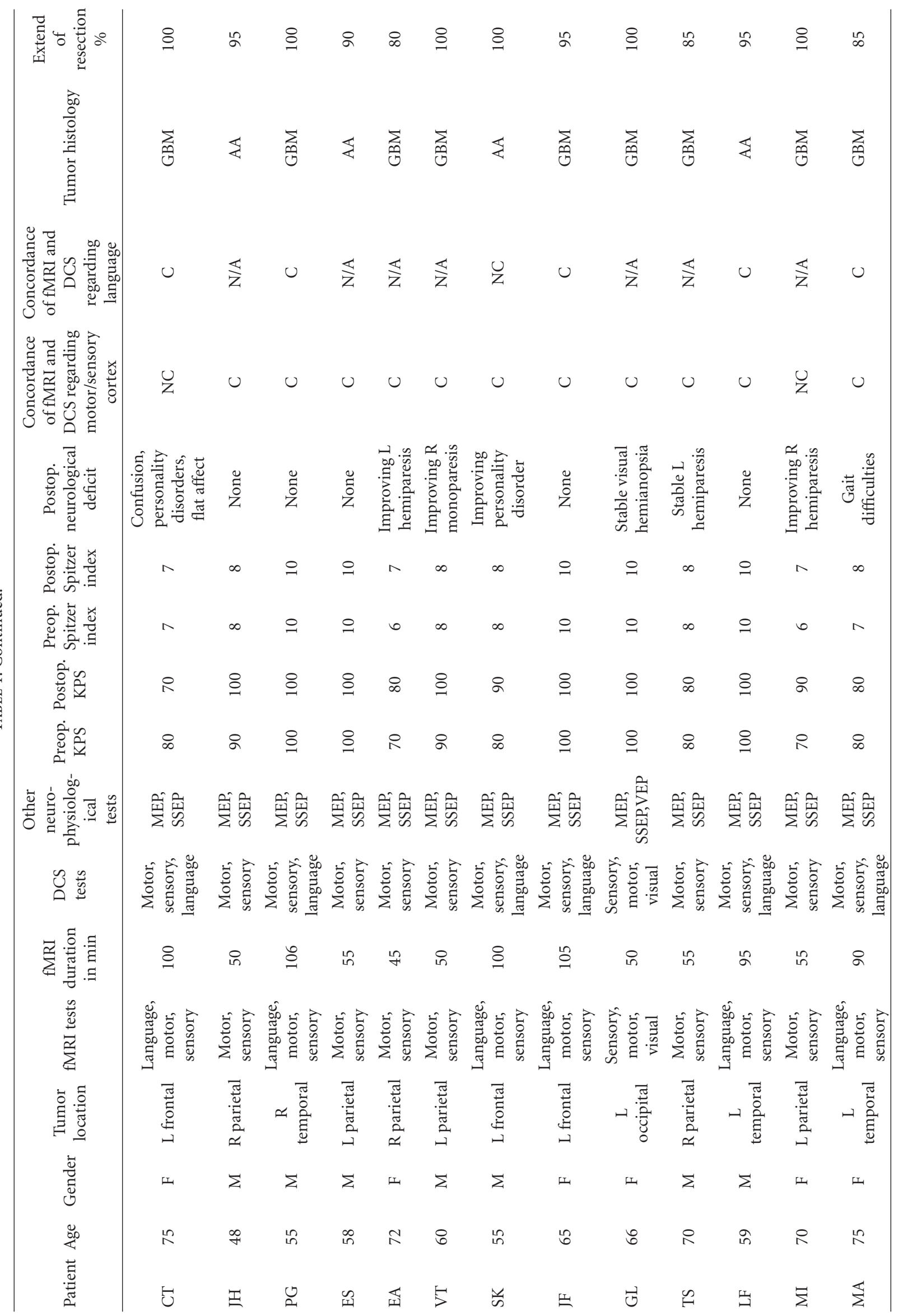




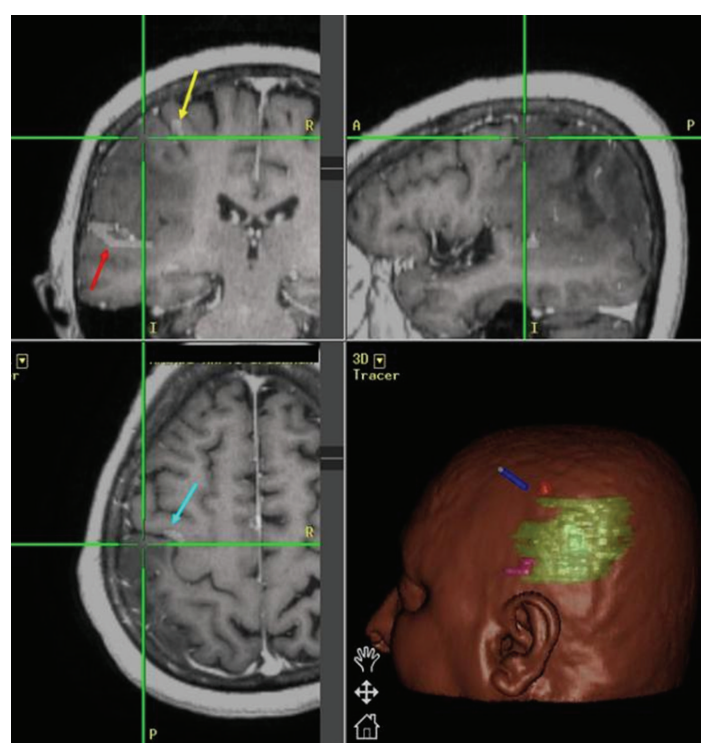

FIGURE 2: Neuronavigation workstation reproduced image showing superimposition of the fMRI data on the MR images. Red arrow shows the language area, yellow arrow shows right-hand motor area, and blue arrow shows ( $\mathrm{R}$ hand) activation just anterior to the central sulcus.

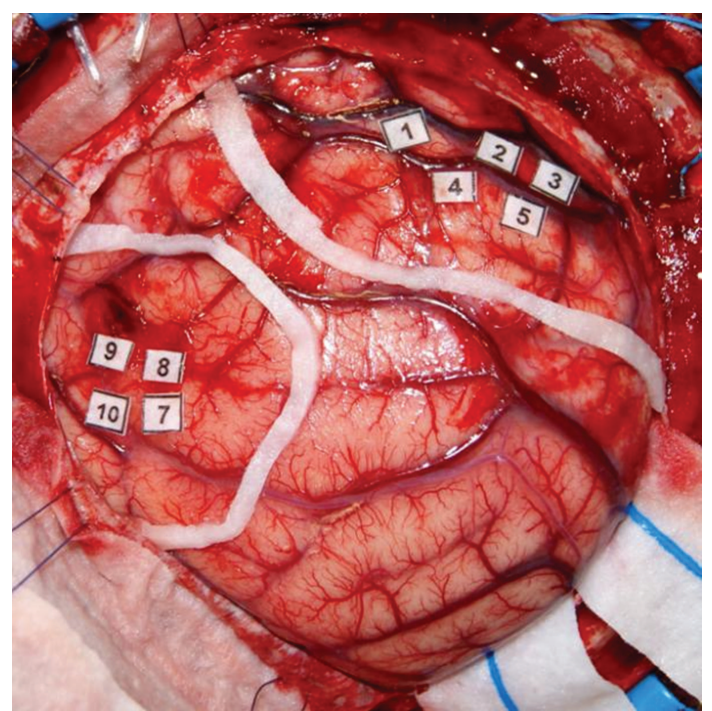

FIGURE 3: Intraoperative photo showing areas of languageassociated activation (stickers 7-10) and right-hand motor activation (stickers 1-5). Note that eloquent areas with $1 \mathrm{~cm}$ safety margin have been outlined by a white cottonoid strap.

on the system's workstation, and image fusion was routinely performed (Figure 2). The accuracy of the system was also tested by identifying well-known anatomical landmarks, such as the osseous nasal bridge, the orbital rim, the medial and lateral canthi, and/or the acoustic meatus.

Intraoperative spontaneous electromyography (EMG), as well as motor, and somatosensory evoked potentials were employed in all our cases. In cases of language mapping awake craniotomy was performed in $31 / 48$ cases $(64.6 \%)$,

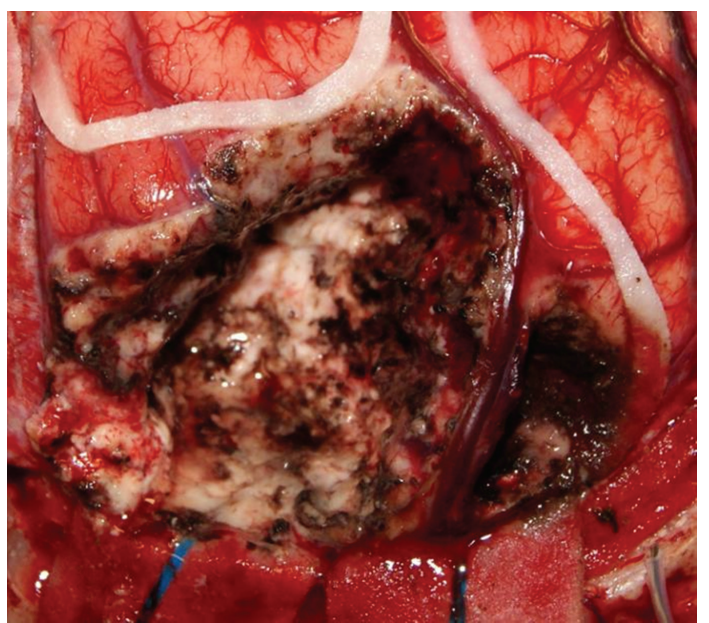

FIGURE 4: Intraoperative photo demonstrating the postresection cavity. The outlined eloquent areas have been preserved as well as the large cortical venous structures in the tumor bed.

while an extraoperative language mapping via previously implanted subdural grid and strip electrodes was performed in the remaining 17 cases (35.4\%). Furthermore, DCS was performed by using an Ojemann bipolar stimulator with constant electric current for stimulation (frequency: $60 \mathrm{~Hz}$, pulse width: $1 \mathrm{msec}$, train: 3 secs, amplitude: $4-12 \mathrm{mAmp}$ ) (Figure 3). Picture naming (flash card demonstration), rehearsing, word listening and parroting, opposite word finding, production of a noun from a verb or vice versa, counting, and countdown from 100 by subtracting 7 were utilized in our language mapping cases. In the case that after-discharges were observed, stimulation was temporarily suspended and the amplitude of the applied stimulus was promptly reduced. Pre and postcentral gyri were successfully identified in all cases, while Broca's and Wernicke's areas in 48/48 patients (Table 1 ).

Special attention was paid during the surgical procedure for avoiding any losses of even minimal amounts of CSF, which could result in brain shift and localization inaccuracies. No osmotic agents or diuretics were used in any of our cases before defining the cortical eloquent areas and outlining the tumor. Moreover, the routine employment of somatosensory and motor evoked potential neuromonitoring confirmed the intraoperative accuracy of the navigator by identifying the central sulcus on each case.

After careful dural opening and prompt identification of the tumor, its contour was outlined on the cortical surface by employing a postfencing technique for which tips of ventricular catheters were utilized for defining the tumor's perimetry. Subpial dissection, aspiration, and resection techniques were used for tumor removal (Figure 4). Furthermore, after completing the tumor resection, sampling biopsies were performed at positions 3, 6, 9, 12 o'clock of the resection cavity for ruling out any further tumor infiltration. 
The findings of fMRI and intraoperative electrophysiologic monitoring regarding the localization of motor, sensory, visual, and language cortical areas were compared. If the fMRI and cortical stimulation areas were exactly the same or less than $5 \mathrm{~mm}$ apart, these studies were considered concordant. In those cases that the distance between the fMRI and the DCS defined eloquent areas was more than $5 \mathrm{~mm}$, the employed studies were considered nonconcordant.

Statistical analysis of our data was performed by employing ANOVA, Fisher, and $\chi^{2}$ statistical tests, while the level of significance was set at $5 \%$. The histopathological reports were recorded in all our cases. An immediate postoperative MRI was obtained (within 72 hours from surgery) for assessing the extent of tumor resection, as well as for establishing a baseline for administrating any further adjuvant treatments. It has to be pointed out that in cases of enhancing lesions removal of the enhancing parts on postoperative MRI was considered as total removal, while in cases of nonenhancing lesions the extent of resection was based on the T2 weighted postoperative images.

\section{Results}

The anatomic location of the tumors in our current series was as follows: (i) 20 patients $(23.0 \%)$ had left frontal tumors, (ii) 25 patients (28.7\%) had left temporal tumors, (iii) 3 patients (3.4\%) had right temporal tumors, (iv) 17 patients (19.5\%) had left parietal tumors, (v) 20 patients (23.0\%) had right parietal tumors, and (vi) 2 patients (2.3\%) had left occipital tumors. Analytical data regarding the patients' demographic data, their tumor anatomic location, their pre and postoperative MMSE and Spitzer index scores are summarized in Table 1.

The mean preoperative KPS score was 91.7 (range: 70-100), while the respective postoperative one was 94.9 (range: 70-100). The mean preoperative Spitzer index was 8.9 (range: $6-10$ ), while the respective postoperative one was 9.1 (range: 6-10). There was worsening of the preoperative KPS score in $2 / 87(2.3 \%)$ of our cases, while the respective percentage for the postoperative Spitzer indices was 1.1\% (1/87 cases). Analysis of our current dataset confirmed that there was a statistically significant association between the patient's age and the tumor histological type $(F$ value $=30.7$, $P$ value $\leq 0.0001)$. Thus, oligodendrogliomas and low grade astrocytomas occurred, as expected in younger patients, while anaplastic astrocytomas in more elderly patients and GBM in even older patients. Contrariwise, there was no statistically significant association between the anatomic location and the tumor histological type in our cohort $\left(\chi^{2}=\right.$ $8.445, P$ value $=0.5854$ ). Moreover, there was a relationship between the patient's age and the occurrence of postoperative neurological deficits. Statistical analysis showed a trend for younger patients to develop less frequently postoperative neurological deficits $(F$ value $=1.627, P$ value $=0.0611)$. A strong relationship was demonstrated between the tumor's anatomic location and the occurrence of postoperative neurological deficits. Patients with tumors located in the right temporal or the occipital lobes developed less frequently postoperative neurological deficits $\left(\chi^{2}=216.993, P\right.$ value $\leq$ $0.0001)$.

In regard to the preoperative fMRI results, the pre and postcentral gyri were identified in all participants. Likewise, Broca's and Wernicke's areas were successfully identified in all our cases requiring language mapping. All tests were performed in the patient's native language (either English or Greek) without testing any additional languages. The visual cortex was successfully identified and was outlined in the obtained fMRI studies in both occipital cases in our series. Intraoperative DCS along with visual evoked potential monitoring confirmed the accuracy of the preoperative fMRI in both cases $(100 \%)$. The mean duration of our fMRI studies was $81.4 \mathrm{~min}$ (range: $45-110 \mathrm{~min}$ ). No complications or adverse effects were observed during the performed fMRI studies.

In regard to the intraoperative DCS, identification of motor and sensory cortical areas was possible in all patients. The identification of the central sulcus was possible in 79/87 cases $(90.8 \%)$ based solely on the recorded SSEPs. The same motor and sensory tasks employed during the obtained fMRI were also utilized during DCS. Similarly, the Broca's and Wernicke's areas were successfully identified in all awake craniotomies, as well as in all extraoperative stimulation and mapping procedures. It has to be mentioned that seizures were elicited during stimulation in four cases (12.9\%) during an awake craniotomy, and in another two cases $(11.8 \%)$ during extraoperative stimulation and mapping studies. All cases were successfully managed with no further consequences for the patient.

The concordance rate of fMRI and intraoperative DCS studies was $91.9 \%$ (80/87 cases) for localizing the sensorymotor cortical areas, while in the remaining $8.1 \%$ the intraoperative electrophysiologic and the fMRI findings were nonconcordant. Statistical analysis of our data revealed that patients with higher preoperative and postoperative KPS scores demonstrated higher percentages of fMRI and DCS concordance $(F$ value $=32.430, P$ value $\leq 0.0001$, and $F$ value $=22.031, P$ value $\leq 0.0001$, resp.). Similarly, patients with high preoperative and postoperative Spitzer index had increased incidence of fMRI and DCS finding concordance in a statistically significant way $(F$ value $=37.893$, $P$ value $\leq 0.0001, F$ value $=26.708, P$ value $\leq 0.0001$, resp.). Furthermore, a statistical trend was established from analyzing our data regarding the tumor's histological type and the concordance of fMRI and DCS findings. It seems that patients harboring GBMs tend to have more frequently nonconcordant fMRI and DCS findings $\left(\chi^{2}=6.983, P\right.$ value $=0.07$ ).

Language-associated cortical areas were accurately outlined by fMRI in $85.4 \%$ (41/48 cases), while in the remaining $14.6 \%$ ( $7 / 48$ cases) the intraoperative electrophysiologic and fMRI findings were nonconcordant. Interestingly, in 5 of these nonconcordant cases, the fMRI localization of the central sulcus and the motor and sensory cortical areas was accurate (Table 1). Statistical analysis of our data showed that patients with higher preoperative and postoperative KPS scores demonstrated higher percentages of fMRI and DCS 
concordance $(F$ value $=20.797, P$ value $\leq 0.0001$, and $F$ value $=6.144, P$ value $=0.0169$, resp. $)$. Similarly, patients with high preoperative and postoperative Spitzer index had increased incidence of fMRI and DCS finding concordance in a statistically significant way $(F$ value $=22.587, P$ value $\leq$ $0.0001, F$ value $=26.831, P$ value $\leq 0.0001$, resp. $)$. However, tumor histological type and grade played no statistically significant role in the observed frequency of fMRI and DCS concordance $\left(\chi^{2}=1.245, P\right.$ value $\left.=0.5366\right)$.

The observed mean extent of tumor resection in our current series was $96.7 \%$ (range: $80-100 \%$ ). Analysis of our data demonstrated no association between the extent of resection and the histological type and grade $(F$ value $=0.234$, $P$ value $=0.8723)$. Interestingly, no relationship could be established in our series between the extent of tumor resection and the occurrence of any postoperative neurological deficits $(F$ value $=1.493, P$ value $=0.1011)$. Furthermore, new postoperative neurological deficits and/or worsening of a preoperative deficit occurred in 26/87 (29.9\%) of our cases. It has to be mentioned, however, that in 14/26 of these patients their postoperative deficits were temporary and their deficits had completely resolved within the first three postoperative months, reducing our actual procedureassociated neurological morbidity to $13.8 \%$ (12/87 patients). Moreover, it has to be emphasized that further neurological improvement was observed among these 12 patients with persistent deficits within the next three months.

The immunohistochemical examination of the resected tumors showed that glioblastoma multiforme occurred in 48 cases (55.2\%), anaplastic astrocytomas in 26 cases $(29.9 \%)$, astrocytomas grade II in 12 patients (13.8\%), and oligodendroglioma grade II in one patient (1.1\%).

\section{Discussion}

Surgical extirpation is theoretically considered the ideal treatment for intracranial gliomas. However, total resection is impossible in the vast majority of cases, mainly due to the infiltrative nature of gliomas, and also due to the presence of eloquent cortex and important neuronal connections in the proximity of intracranial gliomas. It has been clearly demonstrated that intracranial glioma surgical resection reduces their mechanical mass effect on the surrounding brain, diminishes the tumor-associated edema, ensures the establishment of an accurate histological diagnosis, and induces residual tumor cells into active mitotic process, thus making them more vulnerable to any adjuvant postoperative therapy [44, 51, 60-62]. Numerous clinical researchers have shown that extensive glioma resection has been associated with prolonged survival, and better quality of life $[44,51,60-$ 62].

Accurate knowledge of the anatomical relationship of a glioma with its neighboring eloquent cortical areas is of paramount importance for maximizing tumor resection, minimizing the chance of postoperative neurological deficit, and thus maximizing the patient's safety. Functional MRI has been employed for more than a decade in the preoperative evaluation of patients with intracranial gliomas for identifying, and accurately localizing functional cortical networks [6, 27-31, 33, 35-55, 58, 59]. Numerous clinical series have been reported comparing fMRI with intraoperative electrophysiological stimulation studies, with varying study populations, varying tumor histological types, significantly varying fMRI protocols, and thus significantly varying accuracy results, and occasionally contradictory conclusions $[6$, 27-31, 33, 35-55, 58, 59]. Furthermore, a large number of the previously reported series are retrospective studies, carrying all the biases and the weaknesses of retrospective studies. Therefore, the necessity for large-scale, prospective clinical studies comparing the accuracy of fMRI to intraoperative electrophysiological mapping is more than apparent.

Functional MRI accuracy regarding localization of sensory-motor cortical areas was $91.9 \%$ in our cohort. This finding is generally comparable to the existent accuracy rates described recently in the pertinent literature $[6,40$, $41,43,52,59]$. In one of the earliest prospective studies comparing preoperative fMRI (performed at $1.5 \mathrm{~T}$ MRI unit) and DCS for identifying cortical motor areas, Yousry et al. [59] reported a series of six patients with gliomas. They found that the fMRI accuracy in localizing the motor cortex was $100 \%$, when error margin was confined to $10 \mathrm{~mm}$ [59]. Likewise, Mueller et al. [43] reported 100\% fMRI (1.5 T MR unit) accuracy in localizing sensory-motor cortex in their study. At approximately the same time, Schulder et al. [52] reported $100 \%$ accuracy rates for fMRI (performed at $1.5 \mathrm{~T}$ unit) in regard to sensory-motor cortex identification. Lehéricy et al. [40] reported 92\% fMRI (1.5 T MR unit) accuracy in localizing sensory-motor cortex. Li et al. [41] found $100 \%$ fMRI accuracy (performed at $3 \mathrm{~T}$ unit) in outlining the sensory-motor cortex. Recently, Spena et al. [6] reported $92.3 \%$ fMRI (1.5 T unit) accuracy in localizing sensory-motor cortex in their study.

Contrariwise, Atlas et al. [27] reported that were unable to localize by fMRI (performed at $1.5 \mathrm{~T}$ unit) the sensorymotor cortex in $28.5 \%$ of their cases. They postulated that glioblastomas and high-grade gliomas may alter the obtained BOLD signal and make the outlining of the sensory-motor cortex inaccurate or even impossible [27]. Fandino et al. [30] reported only $82 \%$ fMRI ( $1.5 \mathrm{~T}$ unit) accuracy in outlining the sensory-motor cortex. It has to be emphasized, however, that both these clinical series had limited-number study populations.

We found that fMRI was accurate in $85.4 \%$ of our cases in regard to the identification and outlining of the languageassociated cortical areas. These findings are generally in agreement with the previously published experience $[29,31$, $33,42,43,46,49,55,58$ ]. Giussani et al. [33] in a systematic review of the pertinent literature found that fMRI studies could accurately outline language-associated cortical areas in a range of $59-100 \%$. These significantly variable accuracy rates may well be explained by the great variation of the employed fMRI and DCS language paradigms. Additionally, the patient's underlying pathology, the repetition and the confirmation of the language paradigms, as well as the educational level and the cooperation of a patient, may influence both the fMRI and DCS studies. Thus, Mueller et al. [43] reported 100\% fMRI (1.5 T unit) accuracy in 
language-associated cortex outlining. Similarly, Yetkin et al. [58] reported 100\% fMRI (1.5 T unit) accuracy in localizing language-related cortical areas in their series. Likewise, Ruge et al. [49] reported 100\% concordance between fMRI (1.5 T unit) and intraoperative DCS findings in defining language associated cortical areas. FitzGerald et al. [31] reported $81 \%$ sensitivity and $53 \%$ specificity for fMRI (performed at $1.5 \mathrm{~T}$ unit) when the margin error was $10 \mathrm{~mm}$, while the respective percentages were $92 \%$ and $0 \%$ when the margin error was $20 \mathrm{~mm}$. Pouratian et al. [46] reported their experience from employing preoperative fMRI (performed at $3 \mathrm{~T}$ unit) for localizing language-associated cortical areas. Their fMRI sensitivity and specificity rates were $100 \%$ and $66.7 \%$, respectively, for the frontal lobe, while the respective percentages for the temporal and parietal lobes were $96.2 \%$ and $69.8 \%$ [46]. They reported 59\% sensitivity and $97 \%$ specificity for fMRI, and they concluded that fMRI cannot be used alone for surgical planning, in critically-located tumors in language-associated cortical areas [46]. Similarly, Bizzi et al. [29] reported $80 \%$ sensitivity and $78 \%$ specificity for language fMRI (performed at 1.5T unit) in their series. Contrariwise, Lurito et al. [42] reported good but imperfect correlation between fMRI (performed at $1.5 \mathrm{~T}$ unit) and DCS findings regarding language-associated cortical areas. Similarly, Tomczak et al. [55] reported their experience from employing preoperative fMRI (performed at $1.5 \mathrm{~T}$ unit) and intraoperative DCS for outlining language-associated cortical areas in a large glioma series. They found only $33.3 \%$ concordance between the fMRI and DCS findings in their series [55].

Interestingly, analysis of the statistical data of our series showed that in patients with higher preoperative KPS scores and Spitzer indices, fMRI accuracy was higher in a statistically significant way. This may well be explained by the fact that patients with higher KPS and Spitzer scores were more cooperative during fMRI studies and were able to more efficiently execute the utilized sensory, motor, and language fMRI tasks. There was also a statistical trend in observing higher fMRI accuracy in patients with less malignant tumors. This finding may be related to the increased neovascularization of higher grade tumors and the potential alteration of the obtained BOLD signal. This is an important point that needs to be taken into consideration, in order to minimize the chance of inaccuracies and erroneous interpretations of the obtained preoperative fMRI studies.

The concordance rate between AMRI and DCS in regard to the localization of the visual cortex was excellent (100\%) in our series, although the number of occipital cases was very limited (only two patients). Similarly, Hirsch et al. [36] reported their results from a large prospective study of 125 patients with various intracranial pathologies (including, in a large proportion, gliomas), and of 63 healthy volunteers. All their participants underwent fMRI at $1.5 \mathrm{~T}$ unit for sensorymotor, language, and visual cortex localization [36]. They were able to localize in the obtained fMRI the central sulcus in $100 \%$ of their healthy volunteers and in $98.4 \%$ of their patients [36]. They were also able to localize by fMRI the Wernicke's and Broca's areas in $91 \%$ and $77 \%$, respectively, in their patients, while the respective percentages were $100 \%$ and $93 \%$ for their healthy volunteers [36]. The primary visual cortex was identified by fMRI in $100 \%$ of their tested cases [36]. It has to be emphasized, however, that further studying is mandatory before extracting any meaningful conclusions regarding fMRI's accuracy in outlining the visual cortical areas.

The observed mean resection rate in our cohort was $96.7 \%$, while there was no statistically significant association between the extent of resection and the tumor's histological grade. Moreover, no statistically important association was established in our current series between the extent of resection and the occurrence of any new postoperative deficits. It could be postulated that performing fMRI and DCS resulted in more aggressive tumor resection without compromising however the patient's neurological status. Characteristically, worsening of the postoperative KPS scores occurred in only $2.3 \%$ of our patients, while the respective percentage of postoperative Spitzer indices was even lower (1.1\%). Krishnan et al. [39] reported their results from a prospective study including 54 patients with various intracranial tumors. All their patients underwent preoperative fMRI at $1.5 \mathrm{~T}$ and intraoperative DCS and SSEP monitoring for localizing sensory-motor cortical areas [39]. They evaluated their resection rate as well as their morbidity incidence in association with the distance of the resection margin from the fMRI defined motor cortex [39]. They found that when this distance was $>15 \mathrm{~mm}$ their resection rate was $85.7 \%$, while $53 \%$ of their patients remained neurologically unchanged and $47 \%$ were improved postoperatively [39]. When this distance was between 10 $15 \mathrm{~mm}$, total tumor resection was achieved in $86.6 \%$ of their patients, and $13.3 \%$ developed new postoperative neurological deficits, $60 \%$ remained unchanged, while $37.3 \%$ were improved [39]. When the distance was between 5$10 \mathrm{~mm}$, total resection was observed in $83.3 \%$, while $50 \%$ of their patients remained neurologically stable, and the remaining 50\% were improved postoperatively [39]. Finally, when this distance was between $0-5 \mathrm{~mm}$, total resection was accomplished in $85 \%$, neurological worsening occurred in $35 \%$ of their patients, $50 \%$ remained stable, while $15 \%$ were improved [39]. Contrariwise, Berntsen et al. [28] reported significantly lower tumor resection rates in their series. They reported that in $42 \%$ of their cases more than $95 \%$ of the tumor was resected, while their mean residual tumor was $11 \%$ (range: $0-94 \%$ ) [28]. They also reported that $88 \%$ of their patients had stable postoperative neurological status, while $12 \%$ experienced some worsening [28]. Thetumor-to-the-adjacent-eloquent-area distance was related to the amount of tumor residual, and this relationship was statistically significant in their series [28].

Functional MRI provides the opportunity for noninvasively mapping cerebral cortex and outlining functional networks, thus allowing aggressive but safe tumor resection even in cases of close proximity to highly-eloquent areas. However, it carries several weaknesses and has numerous technical limitations. A totally cooperative patient is an absolute requirement since any motion artifacts (head movement, cardiac pulsation, and respiratory movements) may greatly influence its accuracy. Moreover, the educational 
level of the patient may interfere with the fMRI's quality, since many of the tasks, especially those related to language evaluation, require the patient's ability to comprehend and successfully execute them. It also has to be emphasized that fMRI is a time-consuming examination. Additionally, the quality of the obtained fMRI and its accuracy depend on the designing, the characteristics, and the efficacy of the utilized task paradigms every time, and this parameter needs to be taken into consideration during the fMRI interpretation [28].

Furthermore, special glioma-associated conditions may predispose to fMRI inaccuracies. It has been postulated that the presence of pathological neovascularization, particularly in cases of high-grade gliomas, may alter the obtained BOLD signal, and thus may cause false positive activations and fMRI inaccuracies $[27,33,39,53,63]$. It also has been described the induction of cytosol neurochemical changes in high grade gliomas with increased concentrations of nitric oxide as well as altered concentrations of adenosine 59triphosphate, lactate, and glucose, which may influence the obtained BOLD signal and consequently distort the obtained fMRI study [27, 33, 35, 39]. In addition, the presence of tumor-associated arteriovenous shunting, and the presence of tumor-induced edema and mass effect causing mechanical vasoconstriction, as well as the presence of scar tissue secondary to a previous craniotomy, may result into significant BOLD signal changes $[27,33,35,39]$. It has been previously demonstrated that in $10-31 \%$ of the performed fMRI studies the obtained data cannot be processed, while this percentage in solely glioma series ranges between 0-30\% [35]. Moreover, gliomas are usually surrounded by edema and cause mass effect. During the fMRI study, the activation area is located in the brain tissue that may be displaced by the lesion. At the time of the craniotomy, decompression of the brain occurs and alteration of the measured distances between the activation area and the brain tumor may take place. Furthermore, it is well known that fMRI is based on magnetic susceptibility, and thus the presence of a hemorrhage within a brain tumor may alter the accuracy of the BOLD effect and misplace the location of the detected signal on the fMRI. All these parameters have to be taken into consideration for avoiding any fMRI inaccuracies and their potential clinical implications.

The wider clinical application of higher magnetic fields may further increase the quality and consequently the accuracy of the preoperative fMRI. It has been demonstrated that higher magnetic fields may increase the obtained BOLD signal and thus may further improve fMRI's quality and accuracy [33, 37, 38, 41, 54]. Additionally, higher strength magnetic fields allow fMRI studies to be performed in shorter times and provide the opportunity for almost real time imaging of cortical activation during stimulation [64]. Moreover, the development of more concrete paradigms and protocols may further improve fMRI's accuracy and reproducibility. It has been previously demonstrated that loud language tasks provide more accurate fMRI data than silent tasks [45]. Therefore, the development of multistage, loud language tasks examining several aspects of language may further increase fMRI's accuracy. The complementary implementation of other advanced MR imaging techniques such as Diffusion Tensor Imaging (DTI) and intraoperative fMRI and DTI allow better identification and localization not only of the cortical language-associated centers but also their interconnecting networks, and thus make glioma resection safer [65]. Additionally, the wide application of intraoperative MRI and the continuously increasing usage of 5-Amino Levulinic Acid (ALA) and other identifying tumor borders techniques have increased the extent of glioma resection rates, while they have minimized the possibility of any postoperative neurological deficits [6670]. Furthermore, the employment of resting-state fMRI may resolve the BOLD effect alteration caused in gliomas by microvascular and neurochemical cellular pathological changes [71]. These improvements in association with the designing and the development of paradigms and protocols for testing higher cognitive functions such as memory, emotion, executive thinking, and other high-cognitive functions may further increase the applicability of fMRI in the preoperative evaluation of patients with intracranial gliomas.

\section{Conclusions}

Preoperative fMRI successfully identified and outlined motor, sensory, visual, and language-associated cortical networks in all cases in our prospective series. The accuracy of the fMRI was compared to intraoperative electrophysiological mapping via DCS along with evoked potential monitoring. Functional MRI was extremely accurate in localizing motor (91.9\%), sensory (91.9\%), and visual $(100 \%)$ cortical areas, while its accuracy in outlining languageassociated cortical areas was $85.4 \%$. Functional MRI was more accurate in patients with better neurological and functional preoperative status, while it seemed that patients with higher grade gliomas tend to have fMRI inaccuracies more frequently than patients with low grade gliomas. The employment of fMRI along with intraoperative DCS allowed us to achieve an extremely high resection rate (mean: 96.7\%). Worsening of any preoperative neurological deficits and/or occurrence of a new postoperative deficit was observed in $13.8 \%$ of our cases at the completion of the third postoperative month, while worsening of the preoperative functional status occurred in only $2.3 \%$ in our series. The accuracy rate of $\mathrm{fMRI}$ may be further increased by employing solid paradigms, preferably the same paradigms for fMRI and DCS whenever possible, and by ruling out any conditions which could cause fMRI inaccuracies. Although fMRI cannot replace DCS for localizing cortical eloquent areas, when employed in association with DCS can guide and increase DCS's efficacy and provide valuable information for better surgical planning and safer resection of intracranial gliomas.

\section{References}

[1] G. Bertani, E. Fava, G. Casaceli et al., "Intraoperative mapping and monitoring of brain functions for the resection of lowgrade gliomas: technical considerations," Neurosurgical Focus, vol. 27, no. 4, pp. E4.1-E4.16, 2009. 
[2] G. C. Feigl, R. Ritz, M. Moraes et al., "Resection of malignant brain tumors in eloquent cortical areas: a new multimodal approach combining 5-aminolevulinic acid and intraoperative monitoring," Journal of Neurosurgery, vol. 113, no. 2, pp. 352$357,2010$.

[3] S. Gil-Robles and H. Duffau, "Surgical management of World Health Organization Grade II gliomas in eloquent areas: the necessity of preserving a margin around functional structures," Neurosurgical Focus, vol. 28, no. 2, pp. E8.1-E8.9, 2010.

[4] I. L. Maldonado, S. Moritz-Gasser, N. M. De Champfleur, L. Bertram, G. Moulinié, and H. Duffau, "Surgery for gliomas involving the left inferior parietal lobule: new insights into the functional anatomy provided by stimulation mapping in awake patients: clinical article," Journal of Neurosurgery, vol. 115, no. 4, pp. 770-779, 2011.

[5] J. H. Sherman, K. Hoes, J. Marcus, R. J. Komotar, C. W. Brennan, and P. H. Gutin, "Neurosurgery for brain tumors: update on recent technical advances," Current Neurology and Neuroscience Reports, vol. 11, no. 3, pp. 313-319, 2011.

[6] G. Spena, A. Nava, F. Cassini et al., "Preoperative and intraoperative brain mapping for the resection of eloquent-area tumors. A prospective analysis of methodology, correlation, and usefulness based on clinical outcomes," Acta Neurochirurgica, vol. 152, no. 11, pp. 1835-1845, 2010.

[7] J. I. Berman, M. S. Berger, P. Mukherjee, and R. G. Henry, "Diffusion-tensor imaging-guided tracking of fibers of the pyramidal tract combined with intraoperative cortical stimulation mapping in patients with gliomas," Journal of Neurosurgery, vol. 101, no. 1, pp. 66-72, 2004.

[8] A. Quiñones-Hinojosa, S. G. Ojemann, N. Sanai, W. P. Dillon, and M. S. Berger, "Preoperative correlation of intraoperative cortical mapping with magnetic resonance imaging landmarks to predict localization of the Broca area," Journal of Neurosurgery, vol. 99, no. 2, pp. 311-318, 2003.

[9] N. Sanai and M. S. Berger, "Intraoperative stimulation techniques for functional pathway preservation and glioma resection," Neurosurgical Focus, vol. 28, no. 2, pp. E1.1-E1.9, 2010.

[10] J. A. Walker, A. Quiñones-Hinojosa, M. S. Berger, and R. G. Grossman, "Intraoperative speech mapping in 17 bilingual patients undergoing resection of a mass lesion," Neurosurgery, vol. 54, no. 1, pp. 113-118, 2004.

[11] D. Zhang, J. M. Johnston, M. D. Fox et al., "Preoperative sensorimotor mapping in brain tumor patients using spontaneous fluctuations in neuronal activity imaged with functional magnetic resonance imaging: initial experience," Neurosurgery, vol. 65, no. 6, supplement, pp. 226-236, 2009.

[12] A. De Benedictis, S. Moritz-Gasser, and H. Duffau, "Awake mapping optimizes the extent of resection for low-grade gliomas in eloquent areas," Neurosurgery, vol. 66, no. 6, pp. 1074-1084, 2010.

[13] J. Jääskeläinen and T. Randell, "Awake craniotomy in glioma surgery," Acta Neurochirurgica, Supplementum, no. 88, pp. 3135, 2003.

[14] T. Kral, M. Kurthen, J. Schramm, H. Urbach, and B. Meyer, "Stimulation mapping via implanted grid electrodes prior to surgery for gliomas in highly eloquent cortex," Neurosurgery, vol. 58, no. 1, supplement, pp. S-36-S-42, 2006.

[15] R. Schapiro, D. Ferson, S. S. Prabhu, S. Tummula, J. Wefel, and G. Rao, "A technique for mapping cortical areas associated with speech arrest," Stereotactic and Functional Neurosurgery, vol. 90, no. 2, pp. 118-123, 2012.

[16] V. Lubrano, L. Draper, and F. E. Roux, "What makes surgical tumor resection feasible in Broca's area? Insights into intraoperative brain mapping," Neurosurgery, vol. 66, no. 5, pp. 868875,2010 .
[17] A. Szelényi, E. Hattingen, S. Weidauer, V. Seifert, and U. Ziemann, "Intraoperative motor evoked potential alteration in intracranial tumor surgery and its relation to signal alteration in postoperative magnetic resonance imaging," Neurosurgery, vol. 67 , no. 2, pp. 302-313, 2010.

[18] P. A. Bandettini and R. W. Cox, "Event-related fMRI contrast when using constant interstimulus interval: theory and experiment," Magnetic Resonance in Medicine, vol. 43, no. 4, pp. 540$548,2000$.

[19] J. R. Binder, "fMRI of language systems: methods and applications," in Functional MRI-Basic Principles and Applications, S. H. Farro and F. B. Mohamed, Eds., pp. 245-277, Springer Science/Business Media, New York, NY, USA, 2006.

[20] R. M. Birn, P. A. Bandettini, R. W. Cox, and R. Shaker, "Eventrelated fMRI of tasks involving brief motion," Human Brain Mapping, vol. 7, no. 2, pp. 106-114, 1999.

[21] T. Deckersbach, D. D. Doughrty, and S. L. Rauch, "Mood and anxiety disorders," in Functional MRI: Applications in Clinical Neurology and Psychiatry, M. D'Esposito, Ed., pp. 115-135, Informa Healthcare, Oxford, UK, 2006.

[22] J. Hirsch, "Brain mapping for neurosurgery and cognitive neuroscience," in Functional MRI-Basic Principles and Applications, S. H. Farro and F. B. Mohamed, Eds., pp. 39-182, Springer Science/Business Media, New York, NY, USA, 2006.

[23] T. Johnstone, C. M. van Reekum, T. R. Oakes, and R. J. Davidson, "The voice of emotion: an FMRI study of neural responses to angry and happy vocal expressions," Social Cognitive and Affective Neuroscience, vol. 1, no. 3, pp. 242-249, 2006.

[24] B. C. McDonald, A. J. Saykin, J. M. Williams, and B. A. Assaf, "fMRI wada test: prospects for presurgical mapping of language and memory," in Functional MRI-Basic Principles and Applications, S. H. Farro and F. B. Mohamed, Eds., pp. 278-314, Springer Science/Business Media, New York, NY, USA, 2006.

[25] A. Miki, G. T. Liu, and S. H. Faro, "fMRI of the visual pathways," in Functional MRI-Basic Principles and Applications, S. H. Farro and F. B. Mohamed, Eds., pp. 342-363, Springer Science/Business Media, New York, NY, USA, 2006.

[26] E. Z. Kapsalaki, D. Verganelakis, I. Z. Kapsalakis, E. D. Gotsis, and K. N. Fountas, "The role of functional MRI in intracranial glioma resection," in Neuroimaging-Clinical Applications, P. Bright, Ed., pp. 159-172, InTech, Rijeka, Croatia, 2012.

[27] S. W. Atlas, R. S. Howard, J. Maldjian et al., "Functional magnetic resonance imaging of regional brain activity in patients with intracerebral gliomas: findings and implications for clinical management," Neurosurgery, vol. 38, no. 2, pp. 329-338, 1996.

[28] E. M. Berntsen, S. Gulati, O. Solheim et al., "Functional magnetic resonance imaging and diffusion tensor tractography incorporated into an intraoperative 3-dimensional ultrasound-based neuronavigation system: impact on therapeutic strategies, extent of resection, and clinical outcome," Neurosurgery, vol. 67, no. 2, pp. 251-264, 2010.

[29] A. Bizzi, V. Blasi, A. Falini et al., "Presurgical functional MR imaging of language and motor functions: validation with intraoperative electrocortical mapping," Radiology, vol. 248, no. 2, pp. 579-589, 2008.

[30] J. Fandino, S. S. Kollias, H. G. Wieser, A. Valavanis, and Y. Yonekawa, "Intraoperative validation of functional magnetic resonance imaging and cortical reorganization patterns in patients with brain tumors involving the primary motor cortex," Journal of Neurosurgery, vol. 91, no. 2, pp. 238-250, 1999.

[31] D. B. FitzGerald, G. R. Cosgrove, S. Ronner et al., "Location of language in the cortex: a comparison between functional MR 
imaging and electrocortical stimulation," American Journal of Neuroradiology, vol. 18, no. 8, pp. 1529-1539, 1997.

[32] M. T. Forster, E. Hattingen, C. Senft, T. Gasser, V. Seifert, and A. Szelényi, "Navigated transcranial magnetic stimulation and functional magnetic resonance imaging: advanced adjuncts in preoperative planning for central region tumors," Neurosurgery, vol. 68, no. 5, pp. 1317-1324, 2011.

[33] C. Giussani, F. E. Roux, J. Ojemann, E. P. Sganzerla, D. Pirillo, and C. Papagno, "Is preoperative functional magnetic resonance imaging reliable for language areas mapping in brain tumor surgery? Review of language functional magnetic resonance imaging and direct cortical stimulation correlation studies," Neurosurgery, vol. 66, no. 1, pp. 113-120, 2010.

[34] J. M. González-Darder, P. González-López, F. Talamantes et al., "Multimodal navigation in the functional microsurgical resection of intrinsic brain tumors located in eloquent motor areas: role of tractography," Neurosurgical Focus, vol. 28, no. 2, pp. E5.1-E5.10, 2010.

[35] A. Håberg, K. A. Kvistad, G. Unsgård et al., "Preoperative blood oxygen level-dependent functional magnetic resonance imaging in patients with primary brain tumors: clinical application and outcome," Neurosurgery, vol. 54, no. 4, pp. 902-915, 2004.

[36] J. Hirsch, M. I. Ruge, K. H. S. Kim et al., "An integrated functional magnetic resonance imaging procedure for preoperative mapping of cortical areas associated with tactile, motor,language, and visual functions," Neurosurgery, vol. 47, no. 3, pp. 711-722, 2000.

[37] K. Hoenig, C. K. Kuhl, and L. Scheef, "Functional 3.0-T MR assessment of higher cognitive function: are there advantages over 1.5-T imaging?” Radiology, vol. 234, no. 3, pp. 860-868, 2005.

[38] B. Krasnow, L. Tamm, M. D. Greicius et al., "Comparison of fMRI activation at 3 and $1.5 \mathrm{~T}$ during perceptual, cognitive, and affective processing," NeuroImage, vol. 18, no. 4, pp. 813826, 2003.

[39] R. Krishnan, A. Raabe, E. Hattingen et al., "Functional magnetic resonance imaging-integrated neuronavigation: correlation between lesion-to-motor cortex distance and outcome," Neurosurgery, vol. 55, no. 4, pp. 904-915, 2004.

[40] S. Lehéricy, H. Duffau, P. Cornu et al., "Correspondence between functional magnetic resonance imaging somatotopy and individual brain anatomy of the central region: comparison with intraoperative stimulation in patients with brain tumors," Journal of Neurosurgery, vol. 92, no. 4, pp. 589-598, 2000.

[41] S. W. Li, J. F. Wang, T. Jiang et al., "Preoperative 3T high field blood oxygen level dependent functional magnetic resonance imaging for glioma involving sensory cortical areas," Chinese Medical Journal, vol. 123, no. 8, pp. 1006-1010, 2010.

[42] J. T. Lurito, M. J. Lowe, C. Sartorius, and V. P. Mathews, "Comparison of fMRI and intraoperative direct cortical stimulation in localization of receptive language areas," Journal of Computer Assisted Tomography, vol. 24, no. 1, pp. 99-105, 2000.

[43] W. M. Mueller, F. Z. Yetkin, T. A. Hammeke et al., "Functional magnetic resonance imaging mapping of the motor cortex in patients with cerebral tumors," Neurosurgery, vol. 39, no. 3, pp. 515-521, 1996.

[44] National Comprehensive Cancer Network, Central Nervous System Cancer Guidelines, NCCN Press, Jenkintown, Pa, USA, 2007.

[45] N. Petrovich, A. I. Holodny, V. Tabar et al., "Discordance between functional magnetic resonance imaging during silent speech tasks and intraoperative speech arrest," Journal of Neurosurgery, vol. 103, no. 2, pp. 267-274, 2005.

[46] N. Pouratian, S. Y. Bookheimer, D. E. Rex, N. A. Martin, and A. W. Toga, "Utility of preoperative functional magnetic resonance imaging for identifying language cortices in patients with vascular malformations," Neurosurgical Focus, vol. 13, no. 4, article e4, 2002.

[47] A. Puce, R. T. Constable, M. L. Luby et al., "Functional magnetic resonance imaging of sensory and motor cortex: comparison with electrophysiological localization," Journal of Neurosurgery, vol. 83, no. 2, pp. 262-270, 1995.

[48] F. E. Roux, K. Boulanouar, J. A. Lotterie et al., "Language functional magnetic resonance imaging in preoperative assessment of language areas: correlation with direct cortical stimulation," Neurosurgery, vol. 52, no. 6, pp. 1335-1347, 2003.

[49] M. I. Ruge, J. Victor, S. Hosain et al., "Concordance between functional magnetic resonance imaging and intraoperative language mapping," Stereotactic and Functional Neurosurgery, vol. 72, no. 2-4, pp. 95-102, 2000.

[50] G. J. M. Rutten, N. F. Ramsey, P. C. Van Rijen, H. J. Noordmans, and C. W. M. Van Veelen, "Development of a functional magnetic resonance imaging protocol for intraoperative localization of critical temporoparietal language areas," Annals of Neurology, vol. 51, no. 3, pp. 350-360, 2002.

[51] N. Sanai and M. S. Berger, "Glioma extent of resection and its impact on patient outcome," Neurosurgery, vol. 62, no. 4, pp. 753-764, 2008.

[52] M. Schulder, J. A. Maldjian, W. C. Liu et al., "Functional image-guided surgery of intracranial tumors located in or near the sensorimotor cortex," Journal of Neurosurgery, vol. 89, no. 3, pp. 412-418, 1998.

[53] F. Signorelli, J. Guyotat, F. Schneider, J. Isnard, and P. Bret, "Technical refinements for validating functional MRI-based neuronavigation data by electrical stimulation during cortical language mapping," Minimally Invasive Neurosurgery, vol. 46, no. 5, pp. 265-268, 2003.

[54] A. Tieleman, P. Vandemaele, R. Seurinck, K. Deblaere, and E. Achten, "Comparison between functional magnetic resonance imaging at 1.5 and 3 Tesla: effect of increased field strength on 4 paradigms used during presurgical work-up," Investigative Radiology, vol. 42, no. 2, pp. 130-138, 2007.

[55] R. J. Tomczak, A. P. Wunderlich, Y. Wang et al., "fMRI for preoperative neurosurgical mapping of motor cortex and language in a clinical setting," Journal of Computer Assisted Tomography, vol. 24, no. 6, pp. 927-934, 2000.

[56] M. Wengenroth, M. Blatow, J. Guenther, M. Akbar, V. M. Tronnier, and C. Stippich, "Diagnostic benefits of presurgical fMRI in patients with brain tumours in the primary sensorimotor cortex," European Radiology, vol. 21, no. 7, pp. 1517-1525, 2011.

[57] J. M. Wood, B. Kundu, A. Utter et al., "Impact of brain tumor location on morbidity and mortality: a retrospective functional MR imaging study," American Journal of Neuroradiology, vol. 32, no. 8, pp. 1420-1425, 2011.

[58] F. Z. Yetkin, W. M. Mueller, G. L. Morris et al., "Functional MR activation correlated with intraoperative cortical mapping," American Journal of Neuroradiology, vol. 18, no. 7, pp. 13111315, 1997.

[59] T. A. Yousry, U. D. Schmid, A. G. Jassoy et al., "Topography of the cortical motor hand area: prospective study with functional MR imaging and direct motor mapping at surgery," Radiology, vol. 195, no. 1, pp. 23-29, 1995.

[60] M. Lacroix, D. Abi-Said, D. R. Fourney et al., "A multivariate analysis of 416 patients with glioblastoma multiforme: 
prognosis, extent of resection, and survival," Journal of Neurosurgery, vol. 95, no. 2, pp. 190-198, 2001.

[61] J. D. McDonald, B. W. Chong, J. D. Lewine et al., "Integration of preoperative and intraoperative functional brain mapping in a frameless stereotactic environment for lesions near eloquent cortex: technical note," Journal of Neurosurgery, vol. 90, no. 3, pp. 591-598, 1999.

[62] S. L. Stafford, A. Perry, V. J. Suman et al., "Primarily resected meningiomas: outcome and prognostic factors in 581 Mayo Clinic patients, 1978 through 1988," Mayo Clinic Proceedings, vol. 73, no. 10, pp. 936-942, 1998.

[63] J. G. Ojemann, J. M. Neil, A. M. MacLeod et al., "Increased functional vascular response in the region of a glioma," Journal of Cerebral Blood Flow and Metabolism, vol. 18, no. 2, pp. 148153, 1998.

[64] T. Scarabino, G. M. Giannatempo, T. Popolizio et al., "3.0T functional brain imaging: a 5-year experience," Radiologia Medica, vol. 112, no. 1, pp. 97-112, 2007.

[65] C. Nimsky, "Intraoperative acquisition of fMRI and DTI," Neurosurgery Clinics of North America, vol. 22, no. 2, pp. 269277, 2011.

[66] P. M. Black, E. Alexander, C. Martin et al., "Craniotomy for tumor treatment in an intraoperative magnetic resonance imaging unit," Neurosurgery, vol. 45, no. 3, pp. 423-433, 1999.

[67] P. V. Butte, A. N. Mamelak, M. Nuno, S. I. Bannykh, K. L. Black, and L. Marcu, "Fluorescence lifetime spectroscopy for guided therapy of brain tumors," NeuroImage, vol. 54, supplement 1, pp. S125-S135, 2011.

[68] E. B. Claus, A. Horlacher, L. Hsu et al., "Survival rates in patients with low-grade glioma after intraoperative magnetic resonance image guidance," Cancer, vol. 103, no. 6, pp. 12271233, 2005.

[69] M. A. Hatiboglu, J. S. Weinberg, D. Suki et al., "Utilization of intraoperative motor mapping in glioma surgery with highfield intraoperative magnetic resonance imaging," Stereotactic and Functional Neurosurgery, vol. 88, no. 6, pp. 345-352, 2010.

[70] W. Stummer, J. C. Tonn, H. M. Mehdorn et al., "Counterbalancing risks and gains from extended resections in malignant glioma surgery: a supplemental analysis from the randomized 5-aminolevulinic acid glioma resection study: clinical article," Journal of Neurosurgery, vol. 114, no. 3, pp. 613-623, 2011.

[71] J. Martino, S. M. Honma, A. M. Findlay et al., "Resting functional connectivity in patients with brain tumors in eloquent areas," Annals of Neurology, vol. 69, no. 3, pp. 521$532,2011$. 


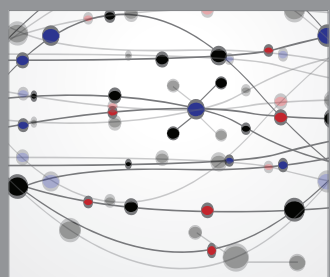

The Scientific World Journal
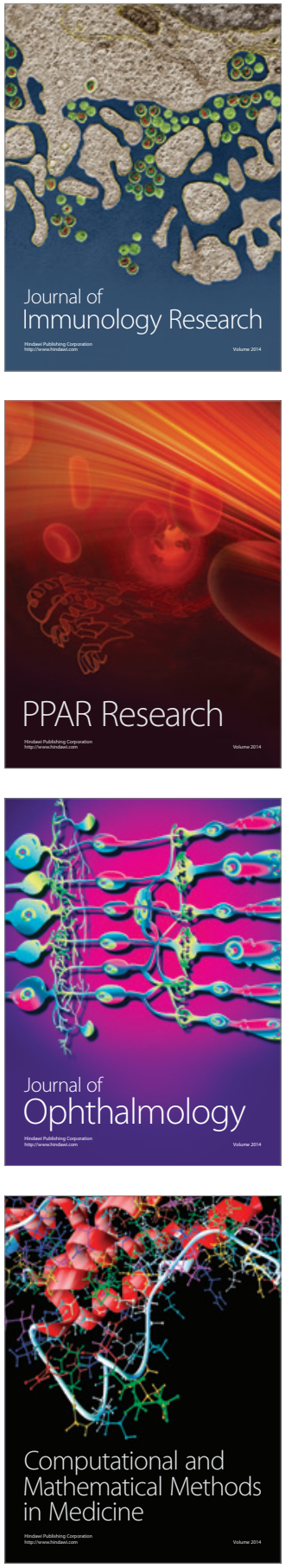

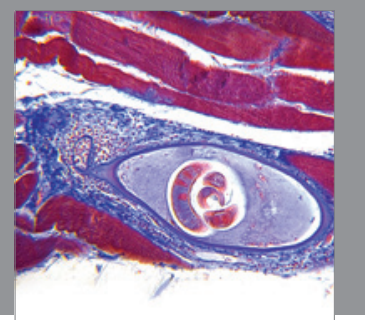

Gastroenterology

Research and Practice
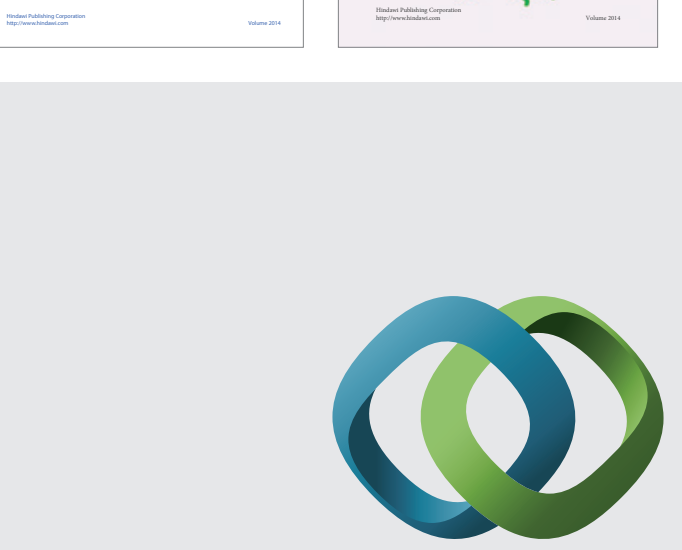

\section{Hindawi}

Submit your manuscripts at

http://www.hindawi.com
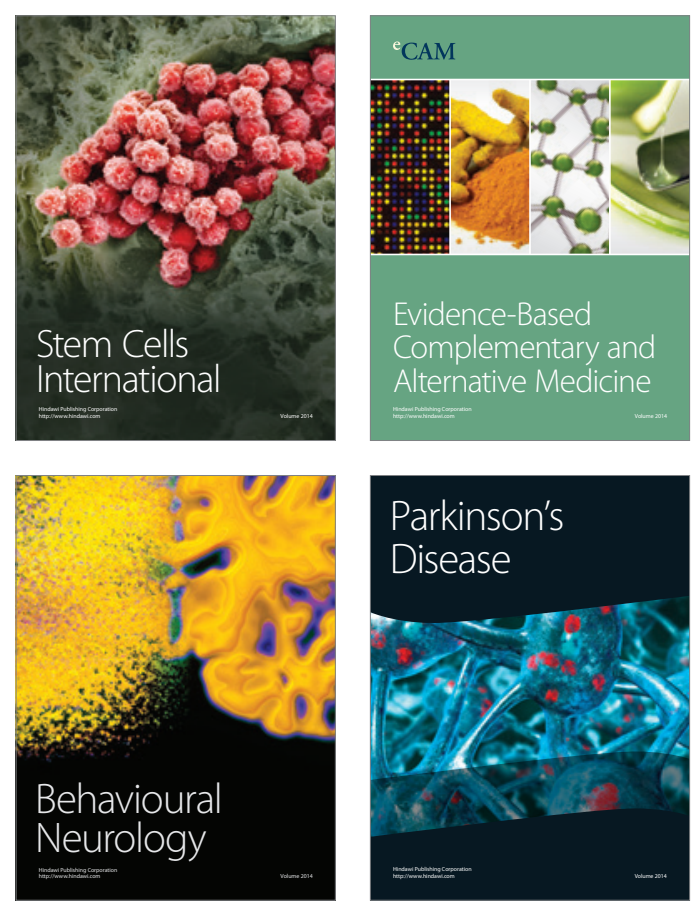

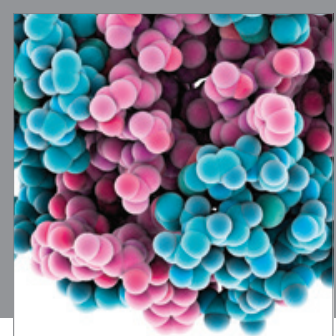

Journal of
Diabetes Research

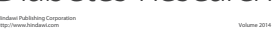

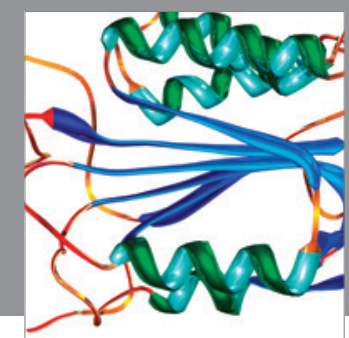

Disease Markers
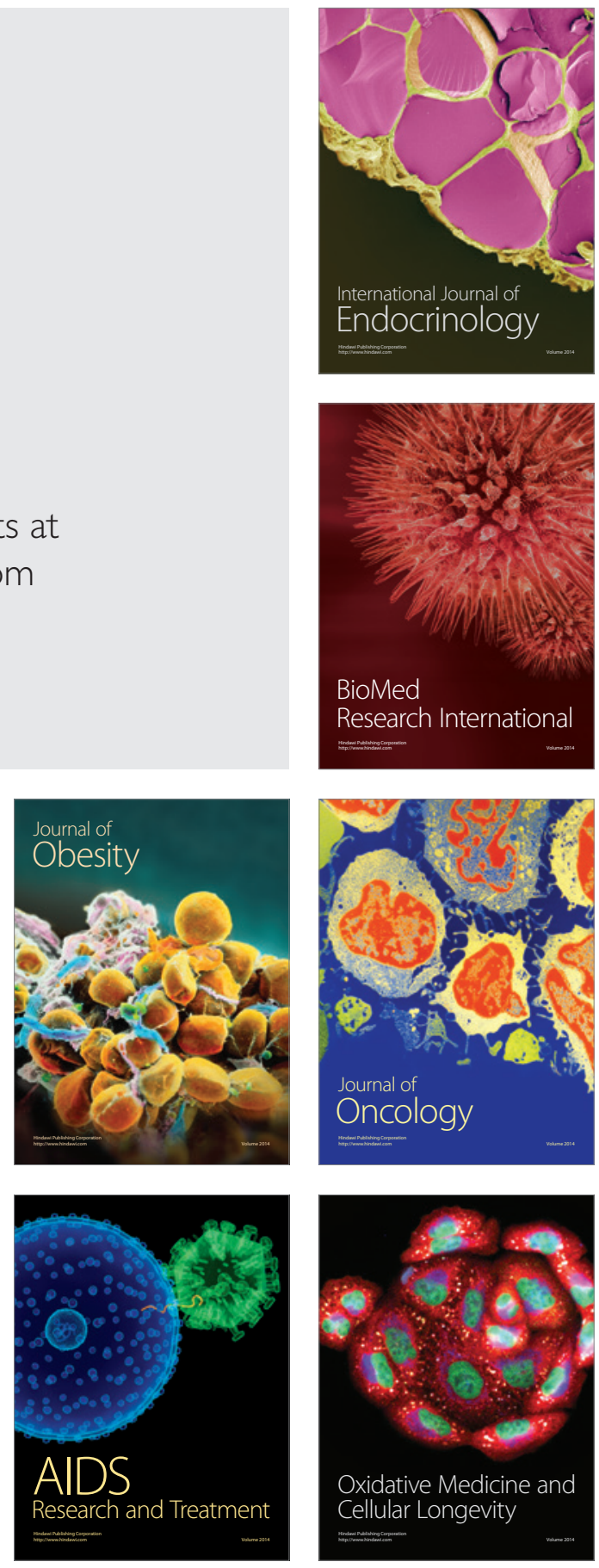